\title{
Continuous local splines of the fourth order of approximation and boundary value problem
}

\author{
I.G.Burova \\ St. Petersburg State University \\ 7/9 Universitetskaya nab., St.Petersburg, 199034 Russia \\ Russia, burovaig@mail.ru, i.g.burova@spbu.ru
}

Received: June 11, 2020, Revised: July 30, 2020. Accepted: August 7, 2020. Published: August 13, 2020.

\begin{abstract}
This paper discusses the construction of polynomial and non-polynomial splines of the fourth order of approximation. The behavior of the Lebesgue constants for the left, the right, and the middle continuous cubic polynomial splines are considered. The non-polynomial splines are used for the construction of the special central difference approximation. The approximation of functions, and the solving of the boundary problem with the polynomial and non-polynomial splines are discussed. Numerical examples are done.
\end{abstract}

Keywords- Polynomial splines, nonpolynomial splines, boundary value problem.

\section{INTRODUCTION}

$\mathrm{R}$ ECENYLY, polynomial and non-polynomial splines are often used to solve a variety of problems: in approximating functions, in constructing numerical schemes for solving boundary value problems, and for solving integral equations (see [1]-[5]). Still a lot of attention is paid to the construction and use of suitable splines in the Galerkin method [6]. At the same time, in the construction and application of splines, little attention is paid to the Lebesgue functions and the Lebesgue constants. The Lebesgue constants are named after Henri Lebesgue. The Lebesgue constants give an idea of how good the approximation of functions are in comparison with the best polynomial approximation of the function (see [7]-[8]).

When constructing interpolation and when studying the quality of the approximation, the authors often use the constant Lebesgue (see [9]-[12]). The exact values of integral Lebesgue constants of generalized special local splines are calculated in [9]. Using the analysis of interpolation error [10], facilitates better node placement for minimizing interpolation error compared to the traditional approach of minimizing the Lebesgue constant as a proxy for interpolation error. The exact values for the uniform Lebesgue constants of interpolating Lsplines are found in [11]. The L-splines, which are bound on the real axis, have equidistant knots and correspond to the linear third-order differential operator. It is proved in [12] that the uniform Lebesgue constant (the norm of a linear operator from
C to C) of local cubic splines with equally spaced nodes, which preserve cubic polynomials, is equal to 11/9.

A spline defect is usually called the difference between the degree of spline and smoothness. Polynomial cubic splines of the maximum defect are used in solving many problems of mathematical physics. Prof. S.G.Mikhlin and prof. Yu.K.Demjanovich have devoted lot of attention to the construction and study of polynomial local splines (see, for example [13]-[14]). In paper [13], methods are given for constructing splines of generalized smoothness in the case of splines of the Lagrangian type on a differentiable manifold. In paper [14], polynomial splines of the Hermitian type are constructed on a uniform grid of nodes.

Earlier, the Lebesgue constant was discussed in connection with the construction of the splines of the maximal defect on a finite grid of nodes in paper [15]. It should be noted that the method of construction of the polynomial coordinate functions proposed in paper [14], as well as the methods of construction of variational-difference equation uses these splines.

Trigonometric splines [16] are often used in solving various problems as they help construct a better result than when we use the polynomial splines.

In this paper we will first dwell briefly on the construction and properties of the polynomial and non-polynomial splines of the fourth order approximation. These non-polynomial splines have the properties of both polynomial and trigonometric splines. Next, we introduce the concepts of functions and Lebesgue constants. Then we consider the use of splines to solve the boundary value problem by the difference method. To construct an algorithm for solving the difference method, we need formulas for the approximate calculation of the first and the second derivatives of the function. These formulas will be constructed on the basis of the non-polynomial splines obtained in this paper. These formulas can be applied to solve boundary value problems. Note that recently the authors have been trying to find alternative approaches for solving differential equations. In paper [18], the numerical solution of nonlinear twodimensional parabolic partial differential equations with initial and Dirichlet boundary conditions is considered. The time 
derivative is approximated using a finite difference scheme whereas space derivatives are approximated using the Haar wavelet collocation method.

\section{The Polynomial CUBIC SPLINES CONSTRUCTION AND LEBESGUE FUNCTIONS}

Let $l, s, m, N$ be non-negative integers such that $l \geq 1, s \geq 1$, $l+s=m+1, N \geq 4$. Let the function $u(x)$ be such that $u \in$ $C^{m+1}([a, b]), a, b$ are real. The nodes $x_{i} \in[a, b], i=0, \ldots, N$, such that $a=x_{0}<\cdots<x_{i-1}<x_{i}<x_{i+1}<\ldots<x_{N}=b$.

The approximation $U(x)$ of function $u(x)$ in the interval $\left[x_{j}, x_{j+1}\right]$ will be constructed in the form

$$
U(x)=\sum_{i} u\left(x_{i}\right) w_{i}(x)
$$

where $w_{i}(x)$ are the interpolation basis polynomial splines which are determined from the conditions:

$$
\sum_{i} \varphi_{\alpha}\left(x_{i}\right) w_{i}(x)=\varphi_{\alpha}(x), \quad \alpha=1, \ldots, m+1 .
$$

The system of functions $\varphi_{\alpha}$ is the Chebyshev system. Here we assume that supp $w_{i}=\left[x_{i-s}, x_{i+l}\right]$. Assuming that the multiplicity of the covering of an arbitrary point $x$ by the supports of basis splines $w_{i}(x)$ is almost everywhere equal to $m+1$. It is easy to see that in sum (1) for $x \in\left[x_{j}, x_{j+1}\right]$ the amount of terms is small:

$$
U(x)=\sum_{i=j-l+1}^{j+s} u\left(x_{i}\right) w_{i}(x)
$$

In this case the basic splines $w_{i}(x)$ are determined from the system of equations uniquely:

$$
\sum_{i=j-l+1}^{j+s} \varphi_{\alpha}\left(x_{i}\right) w_{i}(x)=\varphi_{\alpha}(x), \quad \alpha=1,2, \ldots, m+1
$$

Further, we assume that $\varphi_{1}=1$. With this assumption, the first equation in system (2) has the form:

$$
\sum_{i=j-l+1}^{j+s} w_{i}(x)=1 \text {. }
$$

The splines $w_{i}(x)$ which are constructed in this way have the following properties:

$$
\begin{aligned}
& w_{i}\left(x_{i}\right)=1, w_{i}\left(x_{j}\right)=0, \text { for } j \neq i . \\
& u(x)-U(x)=0 \text { for } u(x)=\varphi_{\alpha}(x), \alpha=1,2, \ldots, m+1 . \\
& w_{j} \in C\left(\left[x_{j-s}, x_{j+l}\right]\right) .
\end{aligned}
$$

Near the boundaries of the finite interval $[a, b]$, we can use either left splines, or right splines. Moreover, to construct an approximation we will only use function values at the grid nodes belonging to the interval $[a, b]$.

As is known, in the case of classical interpolation polynomials, the Lebesgue function and the Lebesgue constant are related to the approximation of a function and the convergence of a sequence of interpolation polynomials to a function.

The error estimation arising due to inaccurate assignment of the function value in the grid nodes can also be investigated using Lebesgue functions.

The Lebesgue function of the basic splines $w_{i}(x)$ with respect to the interval $x \in\left[x_{j}, x_{j+1}\right]$ will be called

$$
\lambda_{j, m+1}(x)=\sum_{i=j-l+1}^{j+s}\left|w_{i}(x)\right|, x \in\left[x_{j}, x_{j+1}\right] .
$$

The Lebesgue constant of the basic splines $w_{i}(x)$ with respect to the interval $\left[x_{j}, x_{j+1}\right]$ will be called the quantity

$$
\lambda_{j, m+1}(x)=\max _{x \in\left[x_{j}, x_{j+1}\right]} \lambda_{j, m+1}(x)
$$

From equation (3) we obtain the inequality $\lambda_{j, m+1}(x) \geq 1$, $x \in\left[x_{j}, x_{j+1}\right]$.

Let $F$ be a real linear normed space of real functions $f(x)$ on the interval $[a, b], n=m+1$. We approximate functions from $F$ by generalized polynomials of the form

$$
\sum_{\alpha=1}^{n} c_{\alpha} \varphi_{\alpha}(x)
$$

Here $c_{k}$ are real numbers.

Let $F_{n}=\operatorname{span}\left\{\varphi_{1}, \ldots, \varphi_{n}\right\}$, where $\varphi_{1}, \ldots, \varphi_{n}$ are the basis functions in $F_{n}$. Consider the function

$$
g\left(c_{1}, \ldots, c_{n}\right)=\left\|u-\sum_{\alpha=1}^{n} c_{\alpha} \varphi_{\alpha}\right\|
$$

The best approximation is the number $d=E_{F_{n}}(u)=\inf _{c_{1}, \ldots, c_{n} \in \mathbb{R}^{n}} g\left(c_{1}, \ldots, c_{n}\right)$. Suppose $d=\|u-\psi\|$. In this case $\psi$ is called the element of the best approximation. In this paper we consider the functions $\varphi_{\alpha}(x)=x^{\alpha-1}, \alpha=$ $1,2,3,4$.

Lemma 1. Let function $u$ be such that $u \in C[a, b]$. The following inequalities are valid:

$$
\begin{gathered}
|U| \leq \lambda_{j, m+1}(x)\|u\|_{C\left[x_{j-l+1}, x_{j+s+1}\right]}, \\
|U| \leq \lambda_{j, m+1}\|u\|_{C\left[x_{j-l+1}, x_{j+s+1}\right]} .
\end{gathered}
$$

Proof. The first inequality follows from the relation

$$
U(x)=\sum_{k=j-l+1}^{j+s} u\left(x_{k}\right) w_{k}(x) .
$$

Calculating the maximum on the right side of this expression when $x \in\left[x_{j}, x_{j+1}\right]$, we get the second relation.

The proof is complete.

Here we discuss similar problems in the case of approximation by polynomial cubic splines. To construct the right, left and middle continuous cubic splines, we need to take $m=3$ and take the parameter $l$, respectively, equal to 1,2 or 3 . 
In the case $l=1$, in the approximation $U$, we use the values of the function $u$ lying to the right of $a$.Thus, we use only grid nodes that only belong to the finite interval $[a, b]$. In the case $l=3$, in the approximation $U$, we use the values of the function $u$ lying to the left of $b$.Thus, we use only grid nodes that belong only to the finite interval $[a, b]$, In the case of $l=2$, the approximation error turns out to be smaller than in the case of $l=1$ or $l=3$. In this case, we need to ensure that in the approximation only grid nodes that are belong to the interval $[a, b]$ are used.

The middle cubic splines give a smaller constant in the approximation error. In the case of a finite interval $[a, b]$, they can be used at intervals spaced from the boundaries at a distance not less than two grid intervals, otherwise, we need the values of the function at the grid nodes that are outside the interval $[a, b]$.

We will use the following notations: $w_{j}^{M}(x), w_{j}^{R}(x), w_{j}^{L}(x)$ are the middle, the right and the left basis cubic polynomial splines, and $\lambda_{4}^{M}, \lambda_{4}^{R}, \lambda_{4}^{L}$ are the Lebesgue constants corresponding to them (we omit the index $j$ ).

The approximation with the right basis splines can be written as follows:

$$
\begin{aligned}
& \quad U^{R}(x)=u\left(x_{j}\right) g_{j}+u\left(x_{j+1}\right) g_{j+1}+u\left(x_{j+2}\right) g_{j+2} \\
& +u\left(x_{j+3}\right) g_{j+3},
\end{aligned}
$$

where

$$
\begin{aligned}
g_{j} & =\frac{\left(x-x_{j+1}\right)\left(x-x_{j+2}\right)\left(x-x_{j+3}\right)}{\left(x_{j}-x_{j+1}\right)\left(x_{j}-x_{j+2}\right)\left(x_{j}-x_{j+3}\right)^{\prime}} \\
g_{j+1} & =\frac{\left(x-x_{j}\right)\left(x-x_{j+2}\right)\left(x-x_{j+3}\right)}{\left(x_{j+1}-x_{j}\right)\left(x_{j+1}-x_{j+2}\right)\left(x_{j+1}-x_{j+3}\right)^{\prime}}, \\
g_{j+2} & =\frac{\left(x-x_{j}\right)\left(x-x_{j+1}\right)\left(x-x_{j+3}\right)}{\left(x_{j+2}-x_{j}\right)\left(x_{j+2}-x_{j+1}\right)\left(x_{j+2}-x_{j+3}\right)^{\prime}} \\
g_{j+3} & =\frac{\left(x-x_{j}\right)\left(x-x_{j+2}\right)\left(x-x_{j+2}\right)}{\left(x_{j+3}-x_{j}\right)\left(x_{j+3}-x_{j+2}\right)\left(x_{j+3}-x_{j+2}\right)} .
\end{aligned}
$$

Lemma 2. Let $N \geq 4, m=3$ and $T(x), x \in\left[x_{j}, x_{j+1}\right]$, be the best polynomial approximation of the function $u(x), u \in$ $C\left(\left[x_{j}, x_{j+3}\right]\right)$. Let $E(u)$ be the best approximation of the function $u(x)$ with $T(x)$. When approximating the function $u(x), x \in\left[x_{j}, x_{j+1}\right]$ with the right basis splines, the inequality is valid:

Proof. We use the relation

$$
\left|U^{R}(x)-u(x)\right| \leq\left(1+\lambda_{4}^{R}\right) E(u) .
$$

$$
U^{R}(x)=\sum_{i=j}^{j+3} u\left(x_{i}\right) w_{i}^{R}(x)
$$

for the approximation $u(x)$ when $x \in\left[x_{j}, x_{j+1}\right]$. Obviously, the relation is valid:

Now we have

$$
T(x)=\sum_{i=j}^{j+3} T\left(x_{i}\right) w_{i}^{R}(x) .
$$

$$
\left|U^{R}(x)-u(x)\right|=\left|U^{R}(x)-T(x)\right|
$$

$$
\begin{aligned}
+|T(x)-u(x)| \leq & E(u)+\left|\sum_{i=j}^{j+3}\left(T\left(x_{i}\right)-u\left(x_{i}\right)\right) w_{i}^{R}(x)\right| \\
& \leq E(u)\left(1+\lambda_{4}^{R}\right) .
\end{aligned}
$$

The proof is complete.

The approximation with the left basis splines can be written as follows:

$$
\begin{gathered}
U^{L}(x)=u\left(x_{j-2}\right) g_{j-2}+u\left(x_{j-1}\right) g_{j-1}+u\left(x_{j}\right) g_{j} \\
+u\left(x_{j+1}\right) g_{j+1},
\end{gathered}
$$

where

$$
\begin{gathered}
g_{j-2}=\frac{\left(x-x_{j-1}\right)\left(x-x_{j}\right)\left(x-x_{j+1}\right)}{\left(x_{j-2}-x_{j-1}\right)\left(x_{j-2}-x_{j}\right)\left(x_{j-2}-x_{j+1}\right)^{\prime}} \\
g_{j-1}=\frac{\left(x-x_{j-2}\right)\left(x-x_{j}\right)\left(x-x_{j+1}\right)}{\left(x_{j-1}-x_{j-2}\right)\left(x_{j-1}-x_{j}\right)\left(x_{j-1}-x_{j+1}\right)^{\prime}} \\
g_{j}=\frac{\left(x-x_{j-2}\right)\left(x-x_{j-1}\right)\left(x-x_{j+1}\right)}{\left(x_{j}-x_{j-2}\right)\left(x_{j}-x_{j-1}\right)\left(x_{j}-x_{j+1}\right)^{\prime}} \\
g_{j+1}=\frac{\left(x-x_{j-2}\right)\left(x-x_{j-1}\right)\left(x-x_{j-2}\right)}{\left(x_{j+1}-x_{j-2}\right)\left(x_{j+1}-x_{j-1}\right)\left(x_{j+1}-x_{j-2}\right)} .
\end{gathered}
$$

Lemma 3. Let $N \geq 4, m=3$ and $T(x), x \in\left[x_{j}, x_{j+1}\right]$, be the best polynomial approximation of the function $u(x), u \in$ $C\left(\left[x_{j-2}, x_{j+1}\right]\right)$. Let $E(u)$ be the best approximation of the function $u(x)$ with $T(x)$.

When approximating the function $u(x), x \in\left[x_{j}, x_{j+1}\right]$ with the left basis splines, the inequality is valid:

$$
\left|U^{L}(x)-u(x)\right| \leq\left(1+\lambda_{4}^{L}\right) E(u) .
$$

Proof. We use the relation

$$
U^{L}(x)=\sum_{i=j-2}^{j+1} u\left(x_{i}\right) w_{i}^{L}(x)
$$

for the approximation $u(x)$ when $x \in\left[x_{j}, x_{j+1}\right]$.

Obviously, the relation is valid:

Now we have

$$
T(x)=\sum_{i=j-2}^{j+1} T\left(x_{i}\right) w_{i}^{L}(x) .
$$

$$
\begin{gathered}
\left|U^{L}(x)-u(x)\right|=\left|U^{L}(x)-T(x)\right|+ \\
|T(x)-u(x)| \leq E(u)+\left|\sum_{i=j-2}^{j+1}\left(T\left(x_{i}\right)-u\left(x_{i}\right)\right) w_{i}^{L}(x)\right| \\
\leq E(u)\left(1+\lambda_{4}^{L}\right) .
\end{gathered}
$$

The proof is complete.

The approximation with the middle basis splines can be written as follows:

$$
\begin{gathered}
U^{M}(x)=u\left(x_{j-1}\right) g_{j-1}+u\left(x_{j}\right) g_{j}+u\left(x_{j+1}\right) g_{j+1} \\
+u\left(x_{j+2}\right) g_{j+2}
\end{gathered}
$$


where

$$
\begin{aligned}
g_{j-1} & =\frac{\left(x-x_{j}\right)\left(x-x_{j+1}\right)\left(x-x_{j+2}\right)}{\left(x_{j-1}-x_{j}\right)\left(x_{j-1}-x_{j+1}\right)\left(x_{j-1}-x_{j+2}\right)^{\prime}} \\
g_{j} & =\frac{\left(x-x_{j-1}\right)\left(x-x_{j+1}\right)\left(x-x_{j+2}\right)}{\left(x_{j}-x_{j-1}\right)\left(x_{j}-x_{j+1}\right)\left(x_{j}-x_{j+2}\right)^{\prime}} \\
g_{j+1} & =\frac{\left(x-x_{j-1}\right)\left(x-x_{j}\right)\left(x-x_{j+2}\right)}{\left(x_{j+1}-x_{j-1}\right)\left(x_{j+1}-x_{j}\right)\left(x_{j+1}-x_{j+2}\right)^{\prime}} \\
g_{j+2} & =\frac{\left(x-x_{j-1}\right)\left(x-x_{j+1}\right)\left(x-x_{j}\right)}{\left(x_{j+2}-x_{j-1}\right)\left(x_{j+2}-x_{j+1}\right)\left(x_{j+2}-x_{j}\right)^{\prime}} .
\end{aligned}
$$

Lemma 4. Let $N \geq 4, m=3$ and $T(x), x \in\left[x_{j}, x_{j+1}\right]$, be the best polynomial approximation of the function $u(x), u \in$ $C\left(\left[x_{j-1}, x_{j+2}\right]\right)$. Let $E(u)$ be the best approximation of the function $u(x)$ with $T(x)$.

When approximating the function $u(x), x \in\left[x_{j}, x_{j+1}\right]$ with the middle basis splines, the inequality is valid:

$$
\left|U^{M}(x)-u(x)\right| \leq\left(1+\lambda_{4}^{M}\right) E(u) .
$$

Proof. We use the relation

$$
U^{M}(x)=\sum_{i=j-1}^{j+2} u\left(x_{i}\right) w_{i}^{M}(x)
$$

for the approximation $u(x)$ when $x \in\left[x_{j}, x_{j+1}\right]$.

Obviously, the relation is valid:

$$
T(x)=\sum_{i=j-1}^{j+2} T\left(x_{i}\right) w_{i}^{M}(x) .
$$

Now we have

$$
\begin{gathered}
\left|U^{M}(x)-u(x)\right|=\left|U^{M}(x)-T(x)\right| \\
+|T(x)-u(x)| \leq E(u)+\left|\sum_{i=j-1}^{j+2}\left(T\left(x_{i}\right)-u\left(x_{i}\right)\right) w_{i}^{M}(x)\right| \\
\leq E(u)\left(1+\lambda_{4}^{M}\right) .
\end{gathered}
$$

The proof is complete.

Let the values of the function $u$ be given with errors, i.e.

$$
V\left(x_{i}\right)=u\left(x_{i}\right)+\varepsilon_{i},
$$

where $\left|\varepsilon_{k}\right| \leq|\varepsilon|$. In this case, instead of

we have

$$
U(x)=\sum_{i=j-l+1}^{j+s} u\left(x_{i}\right) w_{i}(x)
$$

$$
V(x)=\sum_{i=j-l+1}^{j+s}\left(u\left(x_{i}\right)+\varepsilon_{i}\right) w_{i}(x) .
$$

Lemma 5. Let $U\left(x_{i}\right)=u\left(x_{i}\right)+\varepsilon_{i}$, where $\left|\varepsilon_{i}\right| \leq|\varepsilon|$.

Then

$$
|U(x)-V(x)|_{\left[x_{j}, x_{j+1}\right]} \leq \varepsilon \lambda_{m+1} .
$$

Proof. We have

$$
|U(x)-V(x)|_{\left[x_{j}, x_{j+1}\right]} \leq \sum_{i=j-l+1}^{j+s}\left|\varepsilon_{i} w_{i}(x)\right| \leq \varepsilon \lambda_{m+1} .
$$

The proof is complete.

\section{THE CONDENSING GRID OF NODES}

Let us now consider the behavior of the Lebesgue constant on various grids of nodes: condensing according to a certain law and uniform.

We consider an infinite grid of nodes such that

$$
\frac{x_{j+2}-x_{j+1}}{x_{j+1}-x_{j}}=k
$$

First consider the middle basis splines. We have the expression for the Lebesgue function for the middle splines:

$$
\lambda_{4}^{M}(x)=\left|w_{j-1}(x)\right|+\left|w_{j}(x)\right|+\left|w_{j+1}(x)\right|+\left|w_{j+2}(x)\right| .
$$

Replacing $x, x \in\left[x_{j}, x_{j+1}\right]$, by $x=x_{j}+t h, t \in[0,1]$, we obtain

$$
\begin{gathered}
\lambda_{4}^{M}(x)=-\left(-2 t k^{3}+2 t^{2} k^{3}-k^{2}-2 t^{3} k^{2}+2 t^{2} k^{2}-k\right. \\
\left.+2 t k-4 t^{2} k+2 t^{3} k-2 t+2 t^{2}\right) /(k(1+k)) .
\end{gathered}
$$

The maximum of this expression, when $t \in[0,1]$, is reached at the point

$$
t=t_{0}=-\left(-k^{3}+2 k-k^{2}-1+G_{M 1}\right) /(3 k(k-1)),
$$

where $G_{M 1}=\sqrt{k^{6}-k^{5}+k^{3}-k+1}$.

The Lebesgue constant can be written in the form:

$$
\begin{gathered}
\lambda_{4}^{M}=-\left(4 k^{9}-6 k^{8}-12 k^{7}-6 k^{6} G_{M 1}+\right. \\
13 k^{6}+6 k^{5} G_{M 1}+3 k^{5}+3 k^{4}-6 k^{3} G_{M 1}+ \\
13 k^{3}-12 k^{2}-6 k+6 k G_{M 1}+4+2\left(G_{M 1}\right)^{3} \\
\left.-6 G_{M 1}\right) /\left(27(1+k)(k-1)^{2} k^{3}\right) .
\end{gathered}
$$

The graph of $\lambda_{4}^{M}(x)$, when $k$ various from $1 / 2$ to 2 , is shown in Fig.1.

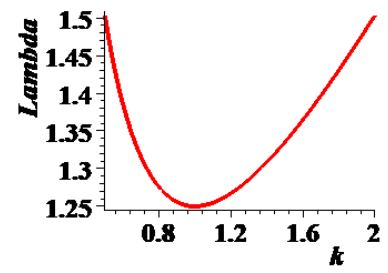

Fig.1. The plot of the $\lambda_{4}^{M}(x)$

We consider the Lebesque constant for the left basis splines:

$$
\lambda_{4}^{L}(x)=\left|w_{j-2}(x)\right|+\left|w_{j-1}(x)\right|+\left|w_{j}(x)\right|+\left|w_{j+1}(x)\right| .
$$

Replacing $x, x \in\left[x_{j}, x_{j+1}\right]$, by $x=x_{j}+t h, t \in[0,1]$, we get

$$
\begin{gathered}
\lambda_{4}^{L}(x)=-\left(2 t^{3} k^{4}-2 t^{2} k^{4}-2 t k^{3}+2 t^{2} k^{3}-2 t k^{2}+\right. \\
\left.2 t^{2} k^{2}-k-1\right) /(1+k) .
\end{gathered}
$$

The maximum of this expression, when $t \in[0,1]$, is reached at the point:

$$
t=t_{0}=\frac{k^{2}-k-1+\sqrt{2 k^{2}+2 k+k^{3}+1+k^{4}}}{3 k^{2}} .
$$

The graph of $\lambda_{4}^{L}$, when $k$ various from $1 / 2$ to 2 , is shown in Fig.2. 


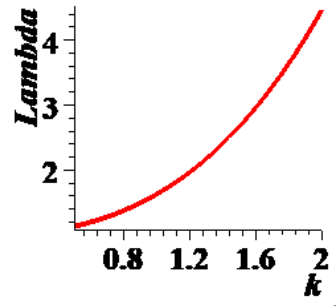

Fig.2. The plot of the $\lambda_{4}^{L}$

The Lebesgue constant can be written in the form:

$$
\begin{gathered}
\lambda_{4}^{L}=-\left(-9 k^{2}-11 k^{3}+12 k-12 G_{L T} k^{2}-\right. \\
12 k G_{L T}-6 k^{4} G_{L T}-6 k^{3} G_{L T}+2\left(G_{L T}\right)^{3}+4- \\
\left.4 k^{6}-6 k^{5}-6 G_{L T}\right) /\left(27 k^{2}(1+k)\right),
\end{gathered}
$$

where

$$
G_{L T}=\sqrt{2 k^{2}+2 k+k^{3}+1+k^{4}} .
$$

Now consider the right basis splines:

$$
\lambda_{4}^{R}(x)=\left|w_{j}(x)\right|+\left|w_{j+1}(x)\right|+\left|w_{j+2}(x)\right|+\left|w_{j+3}(x)\right| .
$$

Replacing $x, x \in\left[x_{j}, x_{j+1}\right]$, by $x=x_{j}+t h, t \in[0,1]$, we get

$$
\begin{gathered}
\lambda_{4}^{R}(x)=k^{4}+k^{3}+2 t k^{2}-2 t^{2} k^{2}+2 t k-2 t^{2} k+ \\
\left.2 t^{3}+2 t-4 t^{2}\right) /\left((1+k) k^{3}\right) .
\end{gathered}
$$

The maximum of this expression, when $t \in[0,1]$, is reached at the point:

where $G=\sqrt{k^{4}+2 k^{3}+2 k^{2}+k+1}$.

$$
t=t_{0}=\frac{k^{2}}{3}+\frac{k}{3}+\frac{2}{3}-G / 3
$$

The Lebesgue constant can be written in the form:

$$
\begin{gathered}
\lambda_{4}^{R}=\left(-4 k^{6}-12 k^{5}+9 k^{4}+4 k^{4} G+8 k^{3} G+\right. \\
\left.11 k^{3}+8 k^{2} G+6 k+4 k G+4 G+4\right) /\left(27(1+k) k^{3}\right) .
\end{gathered}
$$

The graph of $\lambda_{4}^{R}$, when $k$ various from $1 / 2$ to 2 , is shown in Fig.3.

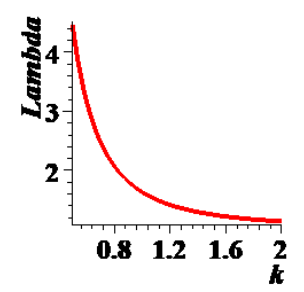

Fig.3. The plot of the $\lambda_{4}^{R}$

The approximation error theorems are easy to formulate.

Theorem 1. Let $k=1$, the grid of nodes is uniform with step $h$. Let $u \in C^{4}([a, b])$.

The following statements are valid:

$$
\begin{array}{ll}
\text { 1) } & \left|u(x)-U^{M}(x)\right| \leq h^{4} \frac{C_{M}}{4 !}\left\|u^{(4)}\right\|_{\left[x_{j-1}, x_{j+2}\right]^{\prime}} \\
\text { 2) } & \left|u(x)-U^{L}(x)\right| \leq h^{4} \frac{C_{L}}{4 !}\left\|u^{(4)}\right\|_{\left[x_{j-2}, x_{j+1}\right]^{\prime}} \\
\text { 3) } & \left|u(x)-U^{R}(x)\right| \leq h^{4} \frac{C_{R}}{4 !}\left\|u^{(4)}\right\|_{\left[x_{j,}, x_{j+3}\right]^{\prime}}
\end{array}
$$

where $C_{L}=C_{R}=1, C_{M}=0.5625$.
Proof. We use the approximation theorem by classical interpolating polynomials. Let $x_{j}=x_{0}+j h, j= \pm 1, \pm 2, \ldots$, and let $x \in\left[x_{j}, x_{j+1}\right]$, be $x=x_{j}+t h, t \in[0,1]$. In order to estimate the error of approximation we use the formula for the remainder of the interpolation polynomial (see [17]). In the case of approximation by the middle basic splines the formula is as follows:

$$
u-U^{M}=\frac{u^{(4)}(\xi(x))}{4 !} \prod_{i=j-1}^{j+2}\left(x-x_{i}\right), \xi(x) \in\left[x_{j-1}, x_{j+2}\right] .
$$

Thus, in the case of approximation by the middle basic splines, it is necessary to find the maximum of the polynomial:

$S_{M}=h^{4}\left|(t-2) t\left(t^{2}-1\right)\right|$. At the point $t=0.5$ we have $\max _{t \in[0,1]} S_{M}=0.5625 h^{4}=C_{M} h^{4}$.

In the case of approximation by the left basic splines, it is necessary to find the maximum of the polynomial:

$S_{L}=h^{4}\left|(t+2) t\left(t^{2}-1\right)\right|$. At the point $t=0.6180340$ we have

$$
\max _{t \in[0,1]} S_{L}=h^{4}=C_{L} h^{4} .
$$

In the case of approximation by the right basic splines, it is necessary to find the maximum of the polynomial:

$S_{R}=h^{4}|(t-2)(t-1)(t-3)(t+2) t|$. At the point $t=$ 0.3819660 we have $\max _{t \in[0,1]} S_{R}=h^{4}=C_{R} h^{4}$.

The proof is complete.

The next example shows that the constants in the statements of the Theorem are valid. Let us take $h=0.3, x_{0}=0, x_{1}=$ $h, x_{2}=2 h, x_{3}=3 h$.

The actual (Act.err.) and theoretical (Theoret.err.) errors of the approximation of functions with the right splines on the interval $h=[0,0.3]$ are given in the Table 1:

Table 1: The actual (Act.err.) and theoretical (Theoret.err.) errors of the approximation of functions with the right splines

\begin{tabular}{|c|l|l|}
\hline Function & Act. err. & Theoret. err. \\
\hline $\sin (3 x)$ & 0.02297 & 0.02767 \\
\hline $\sin (1+2 x)$ & 0.005111 & 0.00540 \\
\hline
\end{tabular}

The calculation results given in Table 1 confirm the correctness of the constants in the approximation estimates of Theorem 1.

\section{The APPLICATION OF THE LEBESGUE FUNCTIONS}

Now we consider a problem. How to select the nodes of the grid so that when using the approximation with the left splines on a one grid interval and using the approximation with the middle splines on the next grid interval then the error of the approximation error was the same in absolute value.

Consider the approximation with the right splines on one grid interval and the approximation with the middle splines on the next grid interval. Solving the equation $\lambda_{4}^{M}(x)=\lambda_{4}^{R}(x)$ we get $k=1.380277569$. We use the approximation with the right splines on the grid interval $\left[x_{j}, x_{j+1}\right]$. We use the approximation with the middle splines on the grid interval $\left[x_{j+1}, x_{j+2}\right]$.

Thus, we obtain the relation $x_{j+2}=x_{j+1}+k\left(x_{j+1}-x_{j}\right)$. 
It follows that the grid step in the approximation with the right splines should be narrower.

The plots of the $\lambda_{4}^{R}$ and $\lambda_{4}^{M}$ are given in Fig.4.

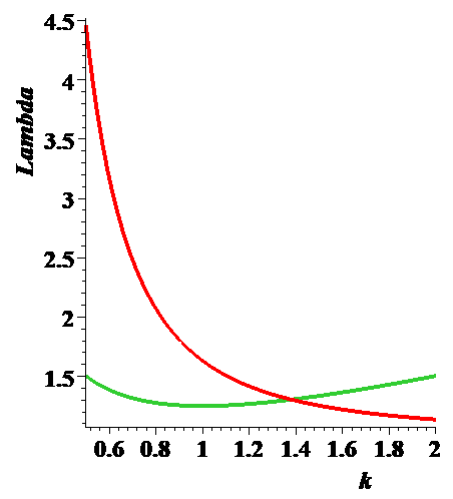

Fig.4. The plot of the $\lambda_{4}^{R}$ and $\lambda_{4}^{M}$

Consider the approximation with the middle splines on one grid interval and the approximation with the left splines on the next grid interval. Solving the equation $\lambda_{4}^{L}=\lambda_{4}^{M}$ we get $k=$ 0.7245.We use the approximation with the middle splines on the grid interval $\left[x_{j}, x_{j+1}\right]$.

We use the approximation with the left splines on the grid interval $\left[x_{j+1}, x_{j+2}\right]$. Thus, we obtain the relation

$$
x_{j+2}=x_{j+1}+k\left(x_{j+1}-x_{j}\right) \text {. }
$$

It follows that the grid step in the approximation with the left splines should be narrower. The plots of the $\lambda_{4}^{L}$ and $\lambda_{4}^{M}$ are given in Fig.5.

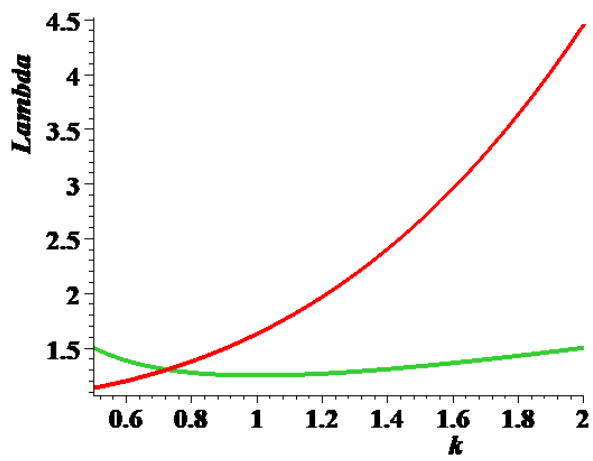

Fig.5. The plot of the $\lambda_{4}^{L}$ and $\lambda_{4}^{M}$

Consider the approximation with the left splines on one grid interval and the approximation with the right splines on the next grid interval. Solving the equation $\lambda_{4}^{R}=\lambda_{4}^{L}$ we get $k=1.0$.

We use the approximation with the right splines on the grid interval $\left[x_{j}, x_{j+1}\right]$. We use the approximation with the left splines on the grid interval $\left[x_{j+1}, x_{j+2}\right]$.

Thus, we obtain the relation

$$
x_{j+2}=x_{j+1}+k\left(x_{j+1}-x_{j}\right) \text {. }
$$

The plots of the $\lambda_{4}^{L}$ and $\lambda_{4}^{R}$ are given in Fig.6.

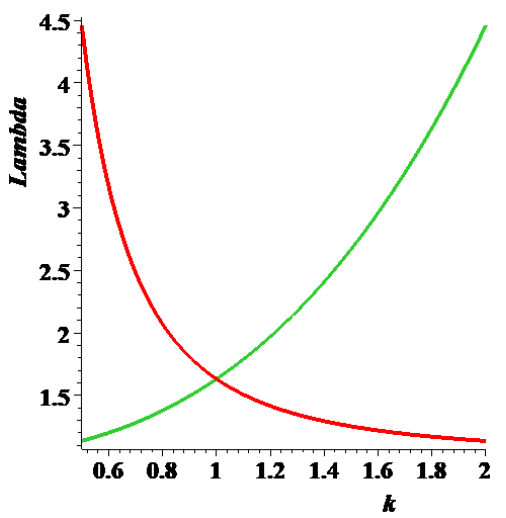

Fig.6. The plot of the $\lambda_{4}^{R}$ and $\lambda_{4}^{L}$

\section{EXAMPLES}

Suppose that the values of the function can be calculated at an arbitrary point in the interval $[a, b]$. How to choose grid nodes so that on each grid interval the approximation error would be approximately the same? Near the left end of the interval $[a, b]$, we apply the right splines. At the following grid intervals we can use the middle splines.

Example 1. We approximate the function $u(x)=\sin (1+$ $2 x)$. First we consider the approximation on a uniform grid of nodes. Let us take $h=0.3, x_{0}=0, x_{1}=h, x_{2}=x_{1}+h=$ $2 h, x_{3}=x_{2}+h=3 h$. The plot of the error of approximation of the function $u(x)=\sin (1+2 x)$ with the right splines on the interval $\left[x_{0}, x_{1}\right]$ and the error of approximation with the middle splines on the interval $\left[x_{1}, x_{2}\right]$ is given in Fig.7 .

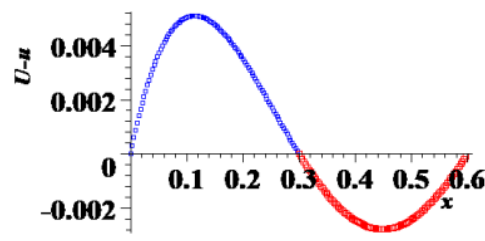

Fig.7. The plot of the error of approximation of the function $u(x)=$ $\sin (1+2 x)$ on the uniform grid of nodes $(h=0.3)$.

Now we move the node $x_{1}$ taking into account the ratio $\frac{x_{2}-x_{1}}{x_{1}-x_{0}}=$ $k, k=1.38$, and construct a grid of nodes such that $x_{0}=$ $0, x_{1}=0.252, x_{2}=0.6, x_{3}=0.948$. On the interval $\left[x_{0}, x_{1}\right]$, we apply the approximation by the right splines, and on the interval $\left[x_{1}, x_{2}\right]$, we apply the approximation by the middle splines. Fig. 8 shows that the errors of the approximation in absolute value with the right and with the middle splines on this non-uniform grid are almost the same.

Let us construct the special non-uniform grid of nodes. We take the first interval $\left[x_{0}, x_{1}\right]$ as before. In the first interval, we apply the approximation by the right splines. In the next interval, which is $\left[x_{1}, x_{2}\right]$, we apply the approximation by the middle splines. To calculate the grid node $x_{2}$, we use the results obtained in the previous section. 


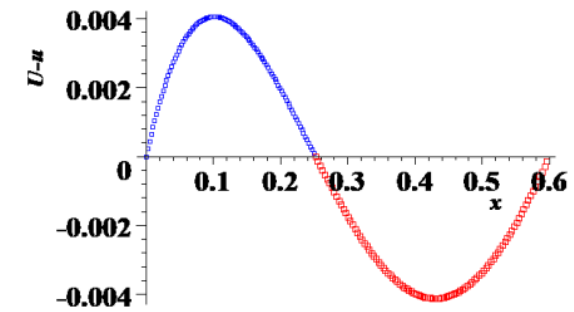

Fig.8. The plot of the error of approximation of the function $u(x)=$ $\sin (1+2 x)$ on the non-uniform grid of nodes.

Let us take $h=0.3 ; k=1.38, x_{0}=0 ; x_{1}=h=0.3 ; x_{2}=$ $x_{1}+k\left(x_{1}-x_{0}\right)=0.714 ; x_{3}=x_{2}+k\left(x_{2}-x_{1}\right)$. The plot of the error of approximation of the function $u(x)=\sin (1+2 x)$ with the right splines on the interval $\left[x_{0}, x_{1}\right]$ and the error of approximation with the middle splines on the interval $\left[x_{1}, x_{2}\right]$ is given in Fig.9.

For comparison, we give a graph of the approximation error with a step $h=0.357$ (Fig.10).

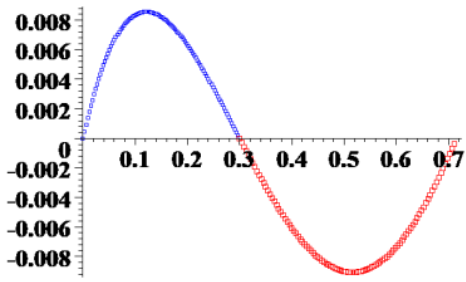

Fig.9. The plots of the error of approximation of the function $u(x)=$ $\sin (1+2 x)$ on the non-uniform grid of nodes.

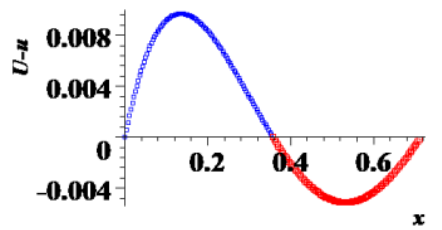

Fig.10. The plot of the error of approximation of the function $u(x)=$ $\sin (1+2 x)$ on the uniform grid of nodes $(h=0.357)$.

\section{ABOUT FORMULAS FOR NUMERICAL DIFFERENTIATION}

The difference method is widely known and is used both for solving ordinary differential equations and for solving partial differential equations. We assume that the solution to the problem exists and is unique. To construct a difference scheme, we first need to construct an approximation of the derivatives. In this section, we will consider the use of non-polynomial splines to construct formulas for numerical differentiation. Note that polynomial and non-polynomial splines can be obtained using the same technique. Since in some cases the approximation by non-polynomial splines gives a smaller approximation error, compared to the approximation by polynomial splines. Therefore, it is hoped that the use of nonpolynomial splines will make it possible to obtain new computational schemes that give a smaller approximation error and are computationally stable.

Thus, to construct an algorithm for solving a boundary value problem with new difference method, we need new numerical differentiation formulas. In addition, it is necessary to establish the stability of the obtained scheme. The stability of the resulting scheme will be discussed later.

The numerical differentiation formulas for the polynomial case are well known. Nevertheless, we show how to obtain formulas for numerical differentiation using the formulas of the middle and left splines.

Differentiating twice the formulas of the middle polynomial splines we get:

$U^{\prime \prime}\left(x_{j}\right)=u_{j-1} g^{\prime \prime}{ }_{j-1}+u_{j} g^{\prime \prime}{ }_{j}+u_{j+1} g^{\prime \prime}{ }_{j+1}+u_{j+2} g^{\prime \prime}{ }_{j+2}$,

where

$$
g^{\prime \prime}{ }_{j-1}=g^{\prime \prime}{ }_{j+1}=\frac{1}{h^{2}}, g^{\prime \prime}{ }_{j}=-\frac{2}{h^{2}}, g^{\prime \prime}{ }_{j+2}=0 .
$$

Note that in the polynomial case on the uniform set of nodes the formulas are well known.

On an uneven grid, formulas for the second derivative are used less frequently. In the polynomial case the formula of the approximation on the non-uniform grid is the next:

$U^{\prime \prime}\left(x_{j}\right)=u_{j-1} g^{\prime \prime}{ }_{j-1}+u_{j} g^{\prime \prime}{ }_{j}+u_{j+1} g^{\prime \prime}{ }_{j+1}+u_{j+2} g^{\prime \prime}{ }_{j+2}$,

where

$g^{\prime \prime}{ }_{j-1}=-2\left(2 x_{j}-x_{j+1}-x_{j+2}\right) / Z n_{0}$

$Z n_{0}=\left(x_{j}-x_{j-1}\right)\left(x_{j-1}-x_{j+1}\right)\left(x_{j-1}-x_{j+2}\right)$,

$g^{\prime \prime}{ }_{j}=2\left(3 x_{j}-x_{j-1}-x_{j+1}-x_{j+2}\right) / Z n$,

$Z n=\left(x_{j}-x_{j+1}\right)\left(x_{j}-x_{j-1}\right)\left(x_{j}-x_{j+2}\right)$,

$g^{\prime \prime}{ }_{j+1}=2\left(2 x_{j}-x_{j-1}-x_{j+2}\right) / Z n_{1}$,

$Z n_{1}=\left(x_{j}-x_{j+1}\right)\left(x_{j-1}-x_{j+1}\right)\left(x_{j-1}-x_{j+2}\right)$,

$g^{\prime \prime}{ }_{j+2}=-2\left(2 x_{j}-x_{j-1}-x_{j+1}\right) / Z n_{2}$,

$Z n_{2}=\left(x_{j}-x_{j+2}\right)\left(x_{j-1}-x_{j+2}\right)\left(x_{j+1}-x_{j+2}\right)$.

Applying the formulas of the left polynomial splines we get:

$U^{\prime \prime}\left(x_{j}\right)=u_{j-1} g^{\prime \prime}{ }_{j-1}+u_{j} g^{\prime \prime}{ }_{j}+u_{j+1} g^{\prime \prime}{ }_{j+1}+u_{j-2} g^{\prime \prime}{ }_{j-2}$,

where

$g^{\prime \prime}{ }_{j-1}=2\left(2 x_{j}-x_{j+1}-x_{j-2}\right) / Z n_{0}$,

$Z n_{0}=\left(x_{j}-x_{j-1}\right)\left(x_{j-2}-x_{j-1}\right)\left(x_{j-1}-x_{j+1}\right)$

$g^{\prime \prime}{ }_{j}=2\left(3 x_{j}-x_{j-1}-x_{j+1}-x_{j-2}\right) / Z n$,

$$
Z n=\left(x_{j}-x_{j+1}\right)\left(x_{j}-x_{j-1}\right)\left(x_{j}-x_{j-2}\right)
$$

$g^{\prime \prime}{ }_{j+1}=-2\left(2 x_{j}-x_{j-1}-x_{j-2}\right) / Z n_{1}$,

$Z n_{1}=\left(x_{j}-x_{j+1}\right)\left(x_{j-2}-x_{j+1}\right)\left(x_{j-1}-x_{j+1}\right)$

$g^{\prime \prime}{ }_{j-2}=-2\left(2 x_{j}-x_{j-1}-x_{j+1}\right) / Z n_{-2}$,

$Z n_{-2}=\left(x_{j}-x_{j-2}\right)\left(x_{j-2}-x_{j-1}\right)\left(x_{j-2}-x_{j+1}\right)$.

We construct new formulas based on the previously obtained non-polynomial splines. Let $\varphi_{1}(x)=1, \varphi_{2}(x)=x, \varphi_{3}(x)=$ $\sin (x), \varphi_{4}(x)=\cos (x)$. We obtain the formula for the middle polynomial-trigonometric (non-polynomial) spline (when $x \in$ $\left.\left[x_{j}, x_{j+1}\right]\right)$ :

$$
\begin{gathered}
U(x)=u\left(x_{j+2}\right) g_{j+2}^{T}+u\left(x_{j-1}\right) g_{j-1}^{T}+u\left(x_{j}\right) g_{j}^{T}(x) \\
\left.+u\left(x_{j+1}\right) g_{j+1}^{T}\right),
\end{gathered}
$$

where

$$
\begin{aligned}
& g_{j}^{T}(x)=\left(( x - x _ { j + 1 } ) \left(\sin \left(x_{j-1}-x_{j+2}\right)\right.\right. \\
& +\left(x_{j-1}-x\right) \sin \left(x_{j+1}-x_{j+2}\right)+\left(x_{j+2}-x\right) \sin \left(x_{j-1}-x_{j+1}\right) \\
& +\left(x_{j+2}-x_{j+1}\right) \sin \left(x-x_{j-1}\right)+\left(x_{j+1}-x_{j-1}\right) \sin \left(x-x_{j+2}\right) \\
& \left.+\left(x_{j-1}-x_{j+2}\right) \sin \left(x-x_{j+1}\right)\right) / \mathrm{Z}
\end{aligned}
$$


where

$$
\begin{aligned}
& Z=\left(x_{j}-x_{j+1}\right) \sin \left(x_{j-1}-x_{j+2}\right)+ \\
& \left(x_{j-1}-x_{j}\right) \sin \left(x_{j+1}-x_{j+2}\right)+ \\
& \left(x_{j+2}-x_{j}\right) \sin \left(x_{j-1}-x_{j+1}\right)+ \\
& \left(x_{j+2}-x_{j+1}\right) \sin \left(x_{j}-x_{j-1}\right)+ \\
& \left(x_{j+1}-x_{j-1}\right) \sin \left(x_{j}-x_{j+2}\right)+ \\
& \left(x_{j-1}-x_{j+2}\right) \text {, } \\
& g_{j+1}^{T}(x)=\left(\left(x_{j}-x\right) \sin \left(x_{j-1}-x_{j+2}\right)+\right. \\
& \left(x_{j+2}-x\right) \sin \left(x_{j}-x_{j-1}\right)+\left(x-x_{j-1}\right) \sin \left(x_{j}-x_{j+2}\right) \\
& +\left(x_{j}-x_{j+2}\right) \sin \left(x-x_{j-1}\right)+\left(x_{j-1}-x_{j}\right) \sin \left(x-x_{j+2}\right) \\
& \left.+\left(x_{j+2}-x_{j-1}\right) \sin \left(x-x_{j}\right)\right) / Z \text {, } \\
& g_{j+2}^{T}(x)=\left(\left(x-x_{j}\right) \sin \left(x_{j-1}-x_{j+1}\right)+\right. \\
& \left(x-x_{j+1}\right) \sin \left(x_{j}-x_{j-1}\right)+\left(x_{j-1}-x\right) \sin \left(x_{j}-x_{j+1}\right)+ \\
& \left(x_{j+1}-x_{j}\right) \sin \left(x-x_{j-1}\right)+\left(x_{j-1}-x_{j+1}\right) \sin \left(x-x_{j}\right)+ \\
& \left.\left(x_{j}-x_{j-1}\right) \sin \left(x-x_{j+1}\right)\right) / Z \text {, } \\
& g_{j-1}^{T}(x)=\left(\left(x-x_{j}\right) \sin \left(x_{j+1}-x_{j+2}\right)+\right. \\
& \left(x-x_{j+2}\right) \sin \left(x_{j}-x_{j+1}\right)+\left(x_{j+1}-x\right) \sin \left(x_{j}-x_{j+2}\right)+ \\
& \left(x_{j}-x_{j+1}\right) \sin \left(x-x_{j+2}\right)+\left(x_{j+2}-x_{j}\right) \sin \left(x-x_{j+1}\right)+ \\
& \left(x_{j+1}-x_{j+2}\right) \sin \left(x-x_{j}\right) / Z \text {. }
\end{aligned}
$$

Based on the middle basis polynomial-trigonometric splines, we obtain the formula:

$$
\begin{gathered}
U^{\prime \prime}\left(x_{j}\right)=U\left(x_{j-1}\right) g^{\prime \prime}{ }_{j-1}+U\left(x_{j}\right) g^{\prime \prime}{ }_{j}+U\left(x_{j+1}\right) g^{\prime \prime}{ }_{j+1} \\
\left.+U\left(x_{j+2}\right) g^{\prime \prime}{ }_{j+2}\right),
\end{gathered}
$$

where

$$
\begin{gathered}
g^{\prime \prime}{ }_{j}=\left(\left(x_{j+1}-x_{j-1}\right) \sin \left(x_{j}-x_{j+2}\right)+\right. \\
\left(x_{j-1}-x_{j+2}\right) \sin \left(x_{j}-x_{j+1}\right)+ \\
\left.\left(x_{j+2}-x_{j+1}\right) \sin \left(x_{j}-x_{j-1}\right)\right) / Z z, \\
g^{\prime \prime}{ }_{j+1}=\left(\left(x_{j}-x_{j+2}\right) \sin \left(x_{j}-x_{j-1}\right)+\right. \\
\left.\left(x_{j-1}-x_{j}\right) \sin \left(x_{j}-x_{j+2}\right)\right) / Z z, \\
Z z=\left(x_{j+1}-x_{j}\right) \sin \left(x_{j-1}-x_{j+2}\right)+ \\
\left(x_{j}-x_{j-1}\right) \sin \left(x_{j+1}-x_{j+2}\right)+ \\
\left(x_{j}-x_{j+2}\right) \sin \left(x_{j-1}-x_{j+1}\right)+ \\
\left(x_{j+1}-x_{j+2}\right) \sin \left(x_{j}-x_{j-1}\right)+ \\
\left(x_{j-1}-x_{j+1}\right) \sin \left(x_{j}-x_{j+2}\right)+ \\
\left(x_{j+2}-x_{j-1}\right) \sin \left(x_{j}-x_{j+1}\right), \\
g^{\prime \prime}{ }_{j+2}=\left(\left(x_{j+1}-x_{j}\right) \sin \left(x_{j}-x_{j-1}\right)+\right. \\
\left.\left(x_{j}-x_{j-1}\right) \sin \left(x_{j}-x_{j+1}\right)\right) / Z z, \\
g_{j-1}^{\prime \prime}=\left(\left(x_{j}-x_{j+1}\right) \sin \left(x_{j}-x_{j+2}\right)+\right. \\
\left.\left(x_{j+2}-x_{j}\right) \sin \left(x_{j}-x_{j+1}\right)\right) / Z z .
\end{gathered}
$$

When $h=$ const we have the formula for the approximating the second derivative of the function:

$U^{\prime \prime}\left(x_{j}\right)=u_{j-1} g^{\prime \prime}{ }_{j-1}+u_{j} g_{j}{ }^{\prime \prime}+u_{j+1} g^{\prime \prime}{ }_{j+1}+u_{j+2} g^{\prime \prime}{ }_{j+2}$, where

$$
\begin{gathered}
g^{\prime \prime}{ }_{j-1}=\frac{2 \sin (h)-\sin (2 h)}{\sin (3 h)+5 \sin (h)-4 \sin (2 h)}, \\
g^{\prime \prime}=\frac{2 \sin (2 h)-4 \sin (2 h)}{\sin (3 h)+5 \sin (h)-4 \sin (2 h)}, \\
g^{\prime \prime}{ }_{j+1}=\frac{2 \sin (h)-\sin (2 h)}{\sin (3 h)+5 \sin (h)-4 \sin (2 h)} . \\
g^{\prime \prime}{ }_{j+2}=0 .
\end{gathered}
$$

Based on the left basis splines, we obtain the formula for the second derivative:

$$
\begin{gathered}
\begin{array}{c}
U^{\prime \prime}\left(x_{j}\right)=\left(U\left(x_{j-2}\right) g^{\prime \prime}{ }_{j-2}+U\left(x_{j-1}\right) g^{\prime \prime}{ }_{j-1}+U\left(x_{j}\right) g^{\prime \prime}{ }_{j}\right. \\
\left.+U\left(x_{j+1}\right) g^{\prime \prime}{ }_{j+1}\right) / Z n
\end{array} \\
\text { where } \\
\begin{array}{c}
g^{\prime \prime}{ }_{j-1}=\left(x_{j}-x_{j+1}\right) \sin \left(x_{j}-x_{j-2}\right)+ \\
\left(x_{j-2}-x_{j}\right) \sin \left(x_{j}-x_{j+1}\right),
\end{array} \\
g^{\prime \prime}{ }_{j-2}=\left(x_{j+1}-x_{j}\right) \sin \left(x_{j}-x_{j-1}\right)+ \\
\left(x_{j}-x_{j-1}\right) \sin \left(x_{j}-x_{j+1}\right), \\
g^{\prime \prime}{ }_{j}=\left(x_{j-2}-x_{j+1}\right) \sin \left(x_{j}-x_{j-1}\right)+ \\
\left(x_{j+1}-x_{j-1}\right) \sin \left(x_{j}-x_{j-2}\right)+ \\
\left(x_{j-1}-x_{j-2}\right) \sin \left(x_{j}-x_{j+1}\right), \\
g_{j+1}^{\prime \prime}=\left(x_{j-1}-x_{j}\right) \sin \left(x_{j}-x_{j-2}\right)+ \\
\left(x_{j}-x_{j-2}\right) \sin \left(x_{j}-x_{j-1}\right), \\
Z n=\left(x_{j}-x_{j+1}\right) \sin \left(x_{j-2}-x_{j-1}\right)+ \\
\left(x_{j-1}-x_{j}\right) \sin \left(x_{j-2}-x_{j+1}\right)+\left(x_{j}-x_{j-2}\right) \sin \left(x_{j-1}-x_{j+1}\right)+ \\
\left(x_{j-1}-x_{j+1}\right) \sin \left(x_{j}-x_{j-2}\right)+\left(x_{j-2}-x_{j-1}\right) \sin \left(x_{j}-x_{j+1}\right)+ \\
\left(x_{j+1}-x_{j-2}\right) \sin \left(x_{j}-x_{j-1}\right) .
\end{gathered}
$$

It can be obtained if the grid of nodes is uniform, then the relation is valid: $u\left(x_{j}\right)=U\left(x_{j}\right)+O\left(h^{2}\right)$.

Nevertheless, we show how to obtain formulas for numerical differentiation using the formulas of the middle and left splines. On the non-uniform set of nodes the formulas for the first derivative such, that $u^{\prime}\left(x_{j}\right)=U^{\prime}\left(x_{j}\right)+O\left(h^{2}\right)$, can be written in the form:

$$
U^{\prime}\left(x_{j}\right)=\left(u\left(x_{j-1}\right) g_{j-1}^{\prime}+u\left(x_{j}\right) g_{j}^{\prime}+u\left(x_{j+1}\right) g_{j+1}^{\prime}\right) / Z n,
$$

where

$$
\begin{gathered}
g_{j-1}^{\prime}=\left(\cos \left(x_{j}-x_{j+1}\right)-1\right) / 2, \\
g_{j+1}^{\prime}=\left(1-\cos \left(x_{j}-x_{j-1}\right)\right) / 2, \\
g_{j}^{\prime}=\left(\cos \left(x_{j}-x_{j-1}\right)-\cos \left(x_{j}-x_{j+1}\right)\right) / 2,
\end{gathered}
$$

$Z n=2 \sin \left(x_{j} / 2-x_{j-1} / 2\right) \sin \left(x_{j-1} / 2-x_{j+1} / 2\right) \sin \left(x_{j} / 2-\right.$

$\left.x_{j+1} / 2\right)$.

On the uniform set of nodes the formula has the form:

$$
u^{\prime}\left(x_{j}\right)=\left(u_{j+1} g_{j+1}^{\prime}-u_{j-1} g_{j-1}^{\prime}\right) /(2 \sin (h))+O\left(h^{2}\right) .
$$


In the polynomial case we have:

$$
U^{\prime}\left(x_{j}\right)=\left(u\left(x_{j-1}\right) g_{j-1}^{\prime}+u\left(x_{j}\right) g_{j}^{\prime}+u\left(x_{j+1}\right) g_{j+1}^{\prime}\right) / Z n
$$

where

$$
\begin{gathered}
g_{j-1}^{\prime}=-\left(x_{j}-x_{j+1}\right)^{2}, \quad g_{j+1}^{\prime}=\left(x_{j}-x_{j-1}\right)^{2}, \\
g_{j}^{\prime}=\left(2 x_{j}-x_{j+1}-x_{j-1}\right)\left(x_{j-1}-x_{j+1}\right), \\
Z n=\left(x_{j}-x_{j-1}\right)\left(x_{j}-x_{j+1}\right)\left(x_{j-1}-x_{j+1}\right) .
\end{gathered}
$$

On the uniform set of nodes we have:

$$
U^{\prime}\left(x_{j}\right)=\left(u\left(x_{j+1}\right)-u\left(x_{j-1}\right)\right) /(2 h) .
$$

We apply the obtained formulas to solve the boundary value problem by the difference method (mesh method).

\section{Example 2. Let us have a problem:}

$$
\begin{gathered}
u^{\prime \prime}+p(x) u^{\prime}-u=f(x), \quad 0 \leq x \leq 1, \\
u(0)=u(1)=0, p(x)=\sin ^{2}(1-x) .
\end{gathered}
$$

We constructed the right side of the equation using the solution of this equation (i.e., the function $u(x)=\sin (x) \sin (x-1)$ ). Thus, we have:

$$
\begin{gathered}
f(x)=\frac{5}{2} \cos (2 x-1)+\frac{1}{2} \sin (2 x-1)-\frac{1}{4} \sin (4 x-3) \\
-\frac{\cos (1)}{2}-\frac{\sin (1)}{4} .
\end{gathered}
$$

The minimum number of internal nodes for using our splines should be 3 . Table 2 shows the results of applying numerical differentiation formulas constructed using polynomial and nonpolynomial splines. It shows the actual errors of the approximate solutions at the grid nodes with the step $h=0.2$, constructed using polynomial and non-polynomial splines. The example shows that in the case of a trigonometric solution, the use of non-polynomial splines takes precedence.

Table 2. The actual errors of the approximate solution obtained using polynomial or non-polynomial splines on a uniform grid of nodes.

\begin{tabular}{|c|l|l|}
\hline$h=0.2$ & Pol.spl. & Non-pol.spl. \\
\hline$x_{1}=h$ & -0.00160 & -0.00120 \\
\hline$x_{2}=2 h$ & -0.00256 & -0.00191 \\
\hline$x_{3}=3 h$ & -0.00261 & -0.00196 \\
\hline$x_{4}=4 h$ & -0.00172 & -0.00129 \\
\hline
\end{tabular}

Let us denote by $U$ the approximate solution that we obtain as a result of solving the problem. The plots of the errors of the approximate solution in absolute value when $n=10$ are given in Figures 11-15. The plot of the errors in absolute value of the approximate solution constructed using non-polynomial splines on the uniform grid of nodes with step $h=0.1$ is given in the Figure 11.

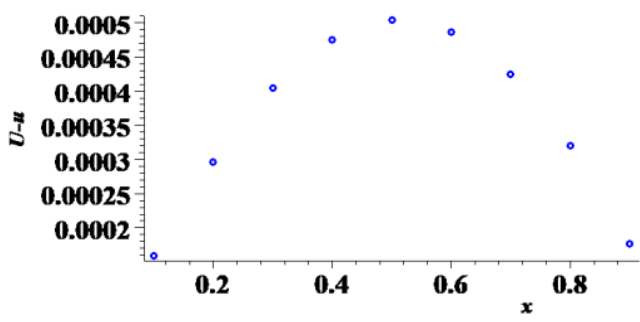

Fig.11. The plot of the errors in absolute values of the approximate solution constructed on the uniform grid of nodes when $n=10$

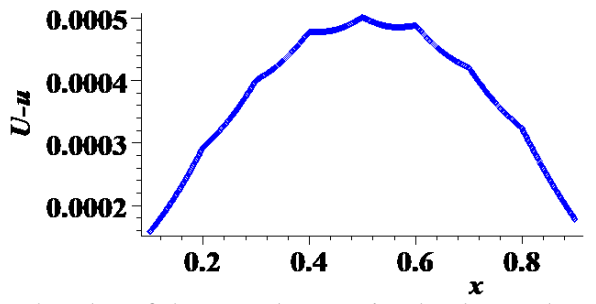

Fig.12. The plot of the actual errors in absolute values of the approximate solution constructed using non-polynomial splines on the uniform grid of nodes when $n=10$

The plot of the errors in absolute value of the approximate solution constructed using polynomial splines on the uniform grid of nodes with step $h=0.1$ is given in Figure 12. Note that in both cases it is easy to the connect points, which were obtained with the difference method, with a line that was constructed by using the formulas of the corresponding spline approximations. Figure 15 shows the points obtained with the difference method and the line which connects the points. We also call the line, which we constructed with the polynomial or non-polynomial splines with the fourth order of approximation, the approximate solution. Figure 12 shows the errors in absolute value of the approximate solution when we constructed the solution between the grid nodes using the formulas of nonpolynomial splines. Figure 14 shows the errors in absolute value of the approximate solution when we constructed the solution between the grid nodes using the formulas of the cubic polynomial splines. Thus, the use of a special non-uniform grid allows us to reduce the error in constructing the solution of the boundary value problem.

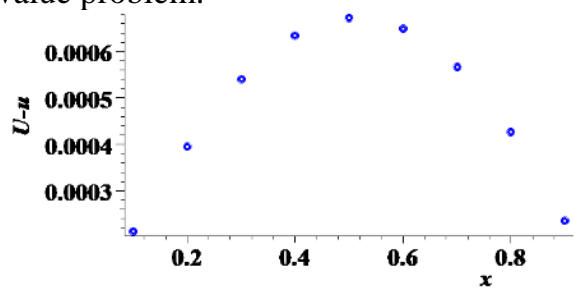

Fig.13. The plot of the errors in absolute values of the approximate solution constructed on the uniform grid of nodes when $n=10$

Now consider an equation with full coefficients. we will solve it first using the traditional method, then using trigonometric approximations.

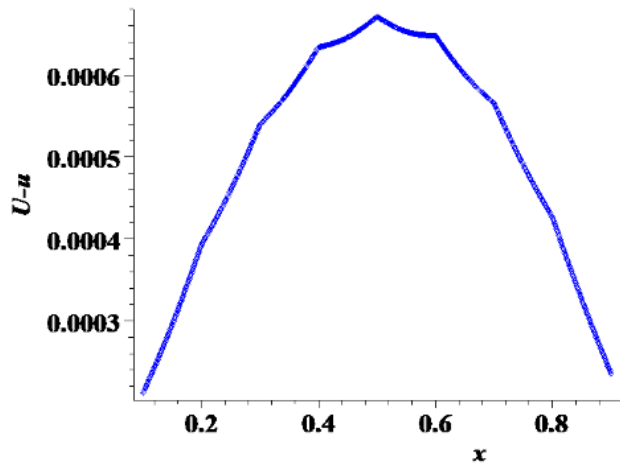

Fig.14. The plot of the actual errors in absolute values of the approximate solution constructed using polynomial splines on the uniform grid of nodes when $n=10$ 


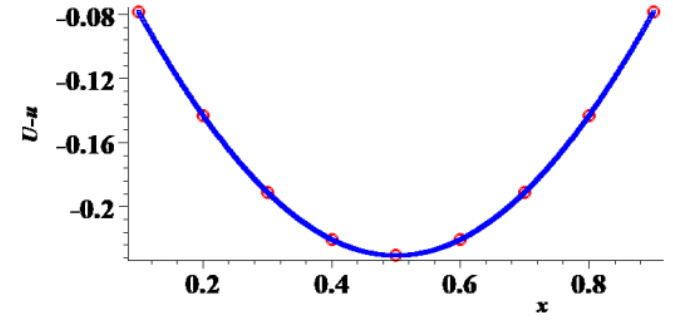

Fig.15. The plot of the approximate solution (red points) and approximate solution constructed using polynomial splines on the uniform grid of nodes when $n=10$

Example 3. Let us have a problem:

$$
\begin{gathered}
u^{\prime \prime}+p(x) u^{\prime}-u=f(x), \quad 0 \leq x \leq 1, \\
u(0)=u(1)=0, \quad p(x)=(1-x)^{2} .
\end{gathered}
$$

We constructed the right side of the equation using the solution of this equation (i.e., the function $\left.u(x)=(x)^{2}(1-x)^{2}\right)$. Thus, we have: $f(x)=2-10 x+x^{2}+20 x^{3}-15 x^{4}+4 x^{5}$.

First, we construct a solution using the usual difference method on a grid of nodes with a step of 0.1 . The error of the solution obtained by the usual difference method (polynomial splines) at the nodes of a uniform grid is presented in the second column of Table 3. The error of the solution obtained using the nonpolynomial splines approximation at the nodes of a uniform grid is presented in the third column of the Table 3. Figure 16 shows a graph of the solution error (in absolute value), obtained using non-polynomial trigonometric splines.

Table 3. The errors of the solution obtained by the usual difference method (Pol.spl.) and using the non-polynomial splines

(Non-pol.spl.)

\begin{tabular}{|c|l|l|}
\hline$h=0.1$ & Pol.spl. & Non-pol.spl. \\
\hline$x_{1}=h$ & -0.000711 & -0.000707 \\
\hline$x_{2}=2 h$ & -0.00131 & -0.00129 \\
\hline$x_{3}=3 h$ & -0.00176 & -0.00173 \\
\hline$x_{4}=4 h$ & -0.00205 & -0.00201 \\
\hline$x_{5}=5 h$ & -0.00216 & -0.00212 \\
\hline$x_{6}=6 h$ & -0.00210 & -0.00206 \\
\hline$x_{7}=7 h$ & -0.00186 & -0.00183 \\
\hline$x_{8}=8 h$ & -0.00143 & -0.00141 \\
\hline$x_{9}=9 h$ & -0.000811 & -0.000805 \\
\hline
\end{tabular}

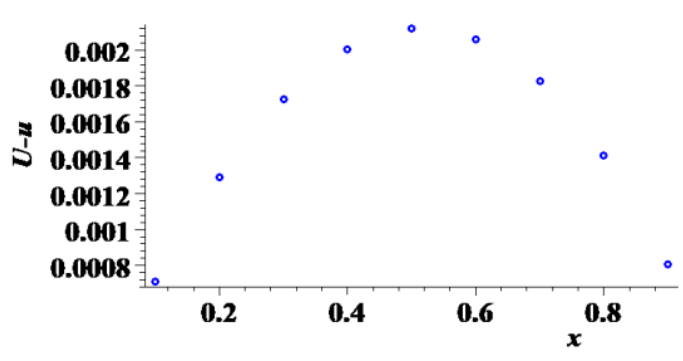

Fig.16. The plot of the errors in absolute values of the approximate solution constructed on the uniform grid of nodes when $n=10$
Examples 2 and 3 show that the use of trigonometric splines to approximate derivatives can improve the error of the resulting solution to the boundary value problem. In the future, it is supposed to consider in more detail the use of other nonpolynomial splines for solving boundary value problems.

\section{ABOUT STABILITY}

The Sturm-Liouville problem $L u=r(x), a \leq x \leq b$, where $L y=-y^{\prime \prime}+p(x) y^{\prime}+q(x) y$, with the simplest boundary conditions $y(a)=\alpha, y(b)=\beta$, was investigated in [17]. In particular, the author of this book noted that if $p, q, r$ are continuous and $q$ positive on $[a, b]$ then the problem has a unique solution. Suppose that there are positive constants $\bar{p}, \underline{q}$, $\bar{q}$ such that $|p(x)| \leq \bar{p}, 0<\underline{q} \leq q(x) \leq \bar{q}$ for $a \leq x \leq b$.

A simple finite difference operator, acting on a grid function $\mathrm{u}$ which approximates the operator $L$ is

$$
\begin{aligned}
& L_{h}=-\frac{u_{j+1}-2 u_{j}+u_{j-1}}{h^{2}}+p\left(x_{j}\right) \frac{u_{j+1}-u_{j-1}}{2 h}+q\left(x_{j}\right) u_{j} \text {, } \\
& j=1,2, \ldots, N \text {. }
\end{aligned}
$$

Theorem 7.3.1 (see [17]) states that if $h \bar{p} \leq 2$, then $L_{h}$ is stable. For non-polynomial finite-difference operators considered in the previous section, a similar statement holds. The proof is similar to that given in the book.

\section{CONCLUSION}

When approximating by the splines of the maximum defect on a finite grid of nodes, it is necessary to use the left, the right, and the middle splines. The right splines are applied near the left end of the interval $[a, b]$, The left splines are applied near the right end of the interval $[a, b]$. The left and the right splines give a large error in absolute value, compared with the middle splines, in the estimation of the approximation error.

Changing the length of the grid intervals, we can make the approximation error in absolute value when using different splines be approximately the same.

In this work, constants are found that make it possible to use the left, the right, or the middle splines at different grid intervals, so that the approximation error in absolute value is the same. The application of information on the behavior of the Lebesgue constants allows us to construct a grid of nodes that allows us to improve the quality of approximation.

In the future, similar theorems will be considered for approximation with the trigonometric splines [16], and the numerical experiment will be discussed in detail. The left, the right and the middle local cubic polynomial splines are conveniently used for approximating functions, including for visualizing the solution of a differential equation. Nonpolynomial local splines of the fourth order of approximation are also conveniently used for approximating functions, including for visualizing the solution of a differential equation. In addition, they allow us to obtain formulas for numerical differentiation with new properties, which can be useful in the construction of difference schemes for solving partial differential equations. This will be the subject of further research. 


\section{References}

[1] R.K.Mohanty, S. Sharma, "Fourth-Order Numerical Scheme Based on Half-Step Non-Polynomial Spline Approximations for 1D Quasi-Linear Parabolic Equations", Numerical Analysis and Applications, 13 (1), 2020, pp. 68-81.

[2] A.Khalid, M.N. Naeem, P.Agarwal, A. Ghaffar, Z. Ullah, S.Jain,

"Numerical approximation for the solution of linear sixth order boundary value problems by cubic B-spline" Advances in Difference Equations,2019(1), 2019, paper №492.

[3] P.K. Srivastava, "A spline-based computational technique applicable for solution of boundary value problem arising in human physiology", International Journal of Computing Science and Mathematics, 10 (1), 2019, pp. 46-57.

[4] R.K.Mohanty, S.Sharma, S.Singh, "A New Two-Level Implicit Scheme for the System of 1D Quasi-Linear Parabolic Partial Differential Equations Using Spline in Compression Approximations", Differential Equations and Dynamical Systems, 27 (1-3), 2019, pp. 327-356.

[5] I.G.Burova, N.S. Domnin, A.E. Vezhlev, A.V. Lebedeva, A.N.Pakulina, "On the solution of the Fredholm equation of the second kind", WSEAS Transactions on Mathematics, 17, 2018, pp. 319-328.

[6] A.Falini, C.Giannelli, T. Kanduč, M.L. Sampoli, A.Sestini, "An adaptive IgA-BEM with hierarchical B-splines based on quasi-interpolation quadrature schemes", International Journal for Numerical Methods in Engineering, 117 (10), 2019, pp. 1038-1058.

[7] Simon J.Smith, "Lebesgue constants in polynomial interpolation," Annales Mathematicae et Informaticae, vol.33, 2006, pp.109-123.

[8] H.-J.Rack, R. Vajda, "On optimal cubic Lagrange interpolation: Extremal node systems with minimal Lebesgue constant," Studia Universitatis Babes-Bolyai Mathematica, vol. 60 (2), 2015, pp. 151-171.

[9] V.T.Shevaldin, "On Integral Lebesgue Constants of Local Splines with Uniform Knots," Proceedings of the Steklov Institute of Mathematics, vol.305, 2019, pp. S158-S165.

[10] D.Moxey, S.P.Sastry, R.M.Kirby, "Interpolation Error Bounds for Curvilinear Finite Elements and Their Implications on Adaptive Mesh Refinement," Journal of Scientific Computing, vol. 78 (2), 2019, pp. 1045-1062.

[11] S.I.Novikov, "Lebesgue Constants for Some Interpolating L-Splines," Proceedings of the Steklov Institute of Mathematics, 300, 2018, pp. 136-144.

[12] V.T.Shevaldin, O.Y.Shevaldina,'Lebesgue Constant of Local Cubic Splines with Equally Spaced Nodes," Numerical Analysis and Applications, 10 (4) , 2017, pp. 362-367.

[13] Yu. K. Dem'yanovich, I. D. Miroshnichenko, E. F. Musafarova, “On Splines' Smoothness," WSEAS Transactions on Mathematics, Vol. 18, 2019, pp. 129-136.

[14] S.G.Mikhlin. "Variational-difference approximation," J. Math. Sci, Vol.10, 1978, pp. 661-787.
[15] I.G.Burova, "On the approximation by quadratic and cubic minimal splines," Numerical Methods, issue 20, 2003, pp. 5-24 (in Russian).

[16] I.G.Burova,"Construction of trigonometric splines", Vestnik St. Petersburg University: Mathematics, vol.37 (2), 2004, pp. 6-11.

[17] Walter Gautschi, "Numerical Analysis. An Introduction", Boston-Basel-Berlin, 1997.

[18] S.Saleem, I.Aziz, M.Z. Hussain, “A simple algorithm for numerical solution of nonlinear parabolic partial differential equations," Engineering with Computers, 2019, Article in Press.

\section{Creative Commons Attribution License 4.0 (Attribution 4.0 International, CC BY 4.0)}

This article is published under the terms of the Creative Commons Attribution License 4.0 https://creativecommons.org/licenses/by/4.0/deed.en_US 